\title{
Prevenção de transtornos alimentares e obesidade: relato de experiência da implementação do programa New Moves
}

\author{
Prevention of eating disorders and obesity: experience report of \\ implementation of the program New Moves
}

Karin Louise Lenz Dunker ${ }^{\mathbf{1}}$, Marle dos Santos Alvarenga², Angélica Medeiros Claudino³

RESUMO O objetivo deste relato foi refletir sobre a adaptação, sobre as dificuldades e sobre as potencialidades de implementação do programa de prevenção conjunta de obesidade e transtornos alimentares New Moves para meninas de escolas públicas de São Paulo (SP). A versão brasileira do programa tem como potencialidades utilizar técnicas comportamentais efetivas, como a teoria sociocognitiva e a entrevista motivacional, e apresentar um conteúdo que aborda fatores comuns à obesidade e aos transtornos alimentares, o qual pode ser aplicado considerando-se as dificuldades quanto à adaptação e implementação apresentadas no estudo.

PALAVRAS-CHAVE Adolescente. Obesidade. Transtornos da alimentação e da ingestão de alimentos. Entrevista motivacional. Saúde pública.

ABSTRACT The aim of this study was to reflect upon the adaptation process, difficulties and potentials of the implementation of the New Moves program, designed to prevent obesity and eating disorders in girls of public schools in São Paulo (SP). The Brazilian version of the program has the potential to use effective behavioral techniques such as socio-cognitive theory and motivational interviewing, and present a content that addresses factors common to obesity and eating disorders, which can be applied considering the difficulties related to adaptation process and implementation presented in the study.

KEYWORDS Adolescent. Obesity. Feeding and eating disorders. Motivational interviewing. Public health.

\footnotetext{
São Paulo (Unifesp) - São Paulo (SP), Brasil.

kdunker00@yahoo.com.br

2 Universidade de São

Paulo (USP) - São Paulo

(SP), Brasil.

marlealvarenga@gmail.com

3 Universidade Federal de São Paulo (Unifesp) - São

Paulo (SP), Brasil.

angelica.claudino@uol.

com.br
}

1 Universidade Federal de 


\section{Introdução}

O programa New Moves (NMB) foi desenvolvido por pesquisadores da Universidade de Minnesota, nos Estados Unidos', com o objetivo de prevenir a obesidade e os Transtornos Alimentares (TA). O foco é a mudança de comportamentos que podem levar à adoção de práticas inadequadas em relação à alimentação, atividade física e aceitação corporal, e não à perda de peso a curto-prazo. Ele foi implementado nas escolas secundárias durante os anos de 2007-2009 com adolescentes do sexo feminino de 14 a 16 anos e teve como resultados principais a diminuição do sedentarismo, melhora no padrão de alimentação, diminuição de comportamentos não saudáveis para controle de peso e melhora na imagem corporal ${ }^{2}$. $\mathrm{O}$ programa foi proposto apenas para meninas de minorias étnicas/raciais e baixo nível socioeconômico por tratar-se de um grupo considerado de risco para o desenvolvimento do sobrepeso/obesidade, menor prática de atividade física, hábitos alimentares inadequados, uso de métodos não saudáveis de controle de peso e insatisfação corporal ${ }^{3,4}$, que também são fatores considerados de risco para o desenvolvimento dos $\mathrm{TA}^{5,6}$. A maioria dos estudos de prevenção dos problemas relacionados com o peso (TA e/ ou obesidade) publicados nas últimas duas décadas com resultados positivos foi aplicada em grupos de adolescentes e estudantes universitários do sexo feminino ${ }^{6}$.

O programa incorpora princípios aprendidos anteriormente em pesquisas da área dos TA e obesidade ${ }^{6,7}$ e está baseado na Teoria Social Cognitiva (TSC), que busca entender porque os indivíduos adquirem ou mantêm comportamentos de saúde específicos ${ }^{8}$. A teoria pressupõe que a mudança da autopercepção dos indivíduos propicia mudanças comportamentais e que, principalmente em adolescentes, a melhora a imagem corporal por meio da promoção de sua autoimagem e de sua autoestima é relevante no tratamento e previne problemas relacionados com o peso e a alimentação ${ }^{9}$. O programa apresenta uma estrutura com conteúdos e dinâmicas que influenciam de forma positiva a prática de atividade física, uma alimentação saudável e a não adoção de métodos de controle de peso inadequados, por meio dos módulos 'Seja Poderosa', 'Seja Ativa' e 'Seja Bem Nutrida' que envolvem conteúdos, respectivamente, de suporte social, atividade física e alimentação.

Segundo Cerin et al. ${ }^{\mathbf{1 0}}$, intervenções que têm como objetivo mudança do comportamento alimentar apresentam melhores resultados se focarem nos construtos da TSC, que incluem: 1) o conhecimento dos riscos e benefícios de diferentes práticas para a saúde; 2) a percepção da autoeficácia, na qual o indivíduo é incentivado a acreditar na sua própria capacidade de mudar um comportamento; 3) as expectativas em relação aos resultados sobre a adoção das práticas - com metas individuais e planejamento de estratégias concretas para incluir ou modificar comportamentos; e 4) a percepção dos facilitadores e impedimentos para realizar as mudanças.

Os construtos da TSC também foram trabalhados com o uso da entrevista motivacional, que é uma técnica diretiva, em que o profissional da saúde guia o indivíduo, por intermédio de um aconselhamento centrado neste, na exploração e na busca das suas próprias motivações para resolver a ambivalência em relação à mudança de comportamentos. É uma abordagem que utiliza ferramentas efetivas na mudança do comportamento, que inclui a escuta reflexiva, aceitação, afirmação e reforço das próprias motivações do indivíduo"11.

É considerada uma técnica eficaz na promoção da atividade física ${ }^{12}$ e na melhora dos comportamentos alimentares ${ }^{13}$. Ela é utilizada para explorar os pensamentos, sentimentos, expectativas e preocupações das adolescentes sobre saúde por meio de um plano de ação quanto aos motivos para a escolha de mudanças relacionadas com os temas do programa. 
Poucos estudos brasileiros de intervenção se baseiam na $\operatorname{TSC}^{\mathbf{1 3}, \mathbf{1 4}}$, sendo que a maioria daqueles que avaliam o efeito no sobrepeso/ obesidade é baseada na educação nutricional 15-17, e poucos investigam os efeitos adversos das intervenções, como o agravamento da estigmatização social ou problemas psicossociais em adolescentes com sobrepeso/ obesidade ou o desenvolvimento dos TA ${ }^{18,19}$.

Estudos que avaliam a efetividade na mudança de comportamentos em relação à alimentação e atividade física, em populações de baixa renda, são, em sua maioria, realizados com populações residentes nos Estados Unidos, potencialmente limitando a generalização dos resultados para populações de países de baixa ou média renda. Tendem a não descrever o conteúdo das intervenções de forma abrangente e o processo de implementação das intervenções, o que traz vários desafios práticos, conceituais e logísticos e torna difícil explorar o que funciona para as pessoas de baixo nível socioeconômico ${ }^{19,20}$.

A discussão sobre as dificuldades e potencialidades de novos programas é essencial para que sejam adaptados para outras populações - de diferentes condições socioeconômicas, culturais e ambientais. Assim, o objetivo deste estudo foi apresentar o processo de implementação do modelo brasileiro do NMB, descrevendo o processo de adaptação do seu conteúdo, as dificuldades e potencialidades no contexto da cidade de São Paulo (SP).

\section{Relato da experiência}

\section{Adaptação do programa New Moves}

O processo de adaptação do programa NMB teve autorização e suporte dos autores originais. Os materiais incluíram: manual das alunas (164 páginas), dinâmicas dos componentes do programa (56 páginas), manual dos profissionais de saúde (82 páginas), roteiro das entrevistas individuais (8 páginas), folhetos para os pais (12 páginas), questionário de avaliação do programa (1 página) e questionário de avaliação das percepções, atitudes e comportamentos relacionados com a alimentação, atividade física e imagem corporal (22 páginas).

Todos os materiais foram traduzidos, adaptados e avaliados por experts na área de nutrição, psicologia e educação física para que o conteúdo fosse de fácil compreensão pelas adolescentes e pais/responsáveis. Os conteúdos das sessões das fases do programa adaptado encontram-se nos quadros 1 e 2, e a descrição das atividades desenvolvidas no programa original e adaptado podem ser acessados em www.newmovesonline.com.

Quadro 1. Conteúdo programático da fase de intervenção do programa New Moves Brasil (NMB)

\begin{tabular}{ll}
\hline Sessão & Atividades desenvolvidas no módulo ‘Seja Ativa' \\
\hline & - Oferecer um ambiente em que as alunas se sentissem confortáveis na prática da atividade física. \\
& - Oferecer atividades físicas de moderadas a vigorosas em, pelo menos, $50 \%$ da aula, e discutir conteúdos \\
ra $9 \quad \begin{array}{l}\text { relacionados com a importância e motivação para a prática da atividade física. } \\
\text { - Aulas realizadas com professor de educação física da equipe de pesquisa: danças, alongamento, treino de } \\
\text { força, jogos cooperativos, circuito. Aulas realizadas por professores convidados: ioga, pilates, capoeira, aeró- } \\
\text { bica, muay thai. }\end{array}$ \\
\hline Sessão & Atividades desenvolvidas no módulo ‘Seja Poderosa' \\
\hline 1 & $\begin{array}{l}\text { É tudo sobre mim: discutir o conceito de autoimagem e criar uma rede de suporte social no grupo que aceita } \\
\text { uma variedade de formas e tamanhos corporais. }\end{array}$
\end{tabular}


Quadro 1. (cont.)

2 Escolha sua aventura: introduzir o conceito de metas, como determiná-las e monitorar as mudanças.

3 Determinando metas que duram: discutir os desafios do uso dos pedômetros e como definir metas relacionadas com a nutrição e atividade física.

$4 \quad$ Um dia na vida: discutir como administrar melhor o tempo para aumentar o nível de atividade física.

$5 \quad$ Mídia: aumentar o conhecimento sobre os fatores que influenciam a imagem corporal e autoimagem. Reconhecer a influência das mensagens irreais apresentadas pela mídia e aprender a avaliar de forma crítica essas mensagens.

$6 \quad$ Menos estresse: identificar sinais de estresse e pensamentos negativos e conhecer técnicas de relaxamento.

$7 \quad$ Armadilha da comparacão: reconhecer os efeitos negativos da comparação e formas de reduzir o hábito de se comparar. Aprender formas de reduzir 'a fala sobre peso'. Identificar características positivas individuais.

$8 \quad$ Finalizando: rever os conteúdos principais apresentados no programa e discutir soluções e estratégias para as barreiras comuns para a mudança da alimentação e atividade física.

\section{Sessão Atividades desenvolvidas no módulo 'Seja Bem Nutrida'}

1 Começando com a nutrição: identificar os benefícios da alimentação saudável. Trabalhar os conceitos de fome, saciedade e tamanho das porções. Discutir a ineficiência do ciclo da dieta.

2 Ahhhh, água refrescante: identificar as razões para beber mais água e limitar o consumo de refrigerantes. Apresentar alternativas para os refrigerantes e bebidas adoçadas.

3 O básico do café da manhã: identificar razões para tomar o café da manhã regularmente. Discutir estratégias para facilitar o consumo do café da manhã. Explorar opções de café da manhã.

$4 \quad$ Explosão das frutas e vegetais: entender a importância do consumo das frutas, verduras e legumes. Identificar formas de aumentar o consumo.

$5 \quad$ Bom para seu lanche: aprender a reconhecer os sinais de fome e saciedade. Identificar lanches saudáveis em situações variadas (ex.: em casa, fora de casa com os amigos, fazendo compras no shopping etc.). Levantar ideias de lanches saudáveis.

6 Vamos almoçar: aprender como realizar um almoço saudável e balanceado. Identificar opções saudáveis de almoço em casa, na escola ou fora de casa

$7 \quad$ Verdades e mitos sobre o fast food: aprender a fazer escolhas saudáveis, mesmo comendo fora de casa. Discutir estratégias para comer fora.

$8 \quad$ Planejando seu futuro: avaliar o progresso durante o programa. Discutir formas de motivação para se manter nas metas.

Quadro 2. Conteúdo programático da fase de manutenção do programa New Moves Brasil (NMB)

\begin{tabular}{ll}
\hline Sessão & Atividades desenvolvidas 'Seja Ativa', 'Seja Poderosa', ‘Seja Bem Nutrida' \\
\hline 1 & Normas socioculturais e padrões de beleza: discutir a influência da mídia nos padrões de beleza. \\
2 & Alimentação e mídia: discutir a influência da mídia sobre as escolhas alimentares. \\
3 & Mídia e atividade física: discutir a influência da mídia sobre saúde e corpo. \\
4 & $\underline{\text { Estresse e emocões: discutir como diferentes formas de estresse podem influenciar no corpo. }}$ \\
5 & Estresse e emoções na alimentaccão: discutir os efeitos do estresse na alimentação, bem como técnicas que \\
6 & Estresse e emoções na atividade física: discutir o uso da prática da atividade física no controle do estresse. \\
7 & $\underline{\text { Influência da família: discutir determinantes genéticos e familiares nas características pessoais. }}$ \\
8 & Família e alimentação: discutir a influência da família nos comportamentos e escolhas alimentares. \\
9 & $\underline{\text { Família e atividade física: discutir a importância da participação da família no incentivo da prática da atividade }}$ \\
\hline
\end{tabular}


Adaptações em relação à duração do NMB ocorreram por conta de diferenças quanto ao calendário escolar e à inserção do programa no currículo escolar. O programa original foi aplicado durante 16 semanas contínuas, como parte do currículo escolar americano; a prática de educação física foi oferecida quatro vezes por semana, e as sessões de suporte social e de nutrição ocorreram de forma intercalada ao longo das 16 semanas, em dias diferentes aos da prática de atividade física.

O calendário escolar brasileiro tem, em média, 18 semanas por semestre, sendo que o ano escolar não é contínuo, pois há dois meses de férias de verão e um mês de férias de inverno. Algumas características da rotina das escolas, como reuniões de planejamento, de pais, recessos escolares por emenda de feriados e a redução da frequência das adolescentes na finalização do semestre, impossibilitaram a realização das 16 semanas propostas no original. Dessa forma, o NMB foi adaptado para 9 semanas (com 17 sessões) na fase intervenção e, após o intervalo de férias, para mais 9 semanas (com 9 sessões) na fase de manutenção. Além da redução do número de semanas, a frequência das aulas de educação física foi de duas vezes por semana, sendo que as sessões de nutrição e suporte social aconteceram semanalmente no mesmo dia - antes das aulas de educação física. O programa aconteceu em horário diferente do escolar, pois, de acordo com as diretrizes governamentais, não poderia ser incluído no currículo.

As sessões foram conduzidas por profissionais da área da saúde treinados. No programa original, os educadores físicos faziam parte do corpo docente e eram treinados para realizar a intervenção - diferentemente do NMB, em que os educadores eram de fora da escola, pois os professores do corpo docente não tinham disponibilidade para $o$ treinamento e aplicação.

Todos os conteúdos teóricos dos módulos foram trabalhados em dinâmicas de grupo, sendo que o 'Seja Ativa' também incluía a atividade prática nos espaços disponíveis (quadras e sala de aula).

\section{Implementação do programa New Moves}

O processo de adaptação dos conteúdos e a aplicação do NMB foram testados inicialmente com 22 adolescentes do sexo feminino de 12 a 14 anos de uma escola pública localizada na região Centro-Sul da cidade de São Paulo (SP) e, após os ajustes, seu impacto foi avaliado em um estudo do tipo ensaio clínico com 270 adolescentes com esse perfil de sexo e idade, em 10 escolas, sendo que 5 receberam a intervenção do NMB e 5 foram consideradas controle.

Os termos de consentimento e assentimento foram assinados pelas adolescentes e seus pais/responsáveis antes da implementação do projeto que foi aprovado pelo Comitê de Ética da Universidade Federal de São Paulo (CAAE:06460112.6.0000.5505). Este estudo foi registrado no Brazilian Registry of Clinical Trials (registration number: RBR-6ddpb3).

Os resultados sobre o efeito do programa indicam que não houve mudanças significativas em relação à satisfação corporal, autoestima, peso e métodos de controle de peso ${ }^{23}$. Os resultados não significativos, indicam a necessidade de relatar as dificuldades e as potencialidades do programa observadas pelos pesquisadores durante sua implementação.

\section{CONTEÚDO}

$\mathrm{Na}$ avaliação da compreensão dos materiais, os ajustes foram quanto aos conteúdos das sessões de nutrição, nos quais os alimentos que faziam parte da cultura americana foram adaptados para a realidade brasileira, em termos de hábitos e condição socioeconômica. Mudanças de sugestões de alimentos de café da manhã foram feitas, como bagel com cream-cheese e uva-passa por bisnaguinha com requeijão; cereal matinal com uva-passa 
por torradas prontas ou feitas em casa; pão ou bagel com manteiga de amendoim por sanduíche de presunto e queijo; burrito com ovos e feijões por tapioca com queijo ou cuscuz com ovos mexidos. Também foram feitas adaptações nos lanches saudáveis (bagel integral por pão de forma integral, pretzel por pipoca do pipoqueiro); frutas, verduras e legumes (frutas ou verduras cortadas em copo por unidades de frutas inteiras); sucos e refrigerantes (bebidas saborizadas light industrializadas por água aromatizada com sabor de frutas e ervas feita em casa); almoço (iogurte com granola, palitos de vegetais, uvas e suco de laranja por omelete de queijo com tomate e ervilha, arroz/torradas e laranja) e alimentos nos restaurantes fast-food cantinas e padarias (smoothie de frutas por vitamina de frutas com iogurte e leite e pretzel por pão na chapa ou misto-quente).

Em relação aos conteúdos das sessões de atividade física, os tipos de atividades indicadas, assim como as realizadas nas aulas práticas, foram adaptados à população de baixa renda, considerando a possibilidade de continuidade dessas práticas em locais de fácil acesso e que ofereçam segurança - como parques, praças e em casa. (troca de ioga na academia por DVD de ioga para prática em casa; patinar por dançar uma música; caminhar ou correr no parque por caminhar no shopping - por questão de segurança).

\section{CONTATO COM AS ESCOLAS}

O contato com as escolas se deu por meio de acesso pessoal dos pesquisadores a cada diretoria de escola. Do total de 46 escolas pertencentes à regional Centro-Sul, que tinham ensino fundamental, de $5^{\mathrm{a}}$ à $8^{\mathrm{a}}$ série, 20 escolas foram consultadas. As 26 escolas restantes foram excluídas por serem de ensino integral, por seu período escolar ser vespertino ou por dificuldade de acesso dos pesquisadores. Observou-se, no processo de adesão das escolas ao projeto de pesquisa, que os motivos de recusa de algumas escolas em participar foram: não ter espaço para a realização das atividades do programa; não ter interesse na proposta por conta de mudanças de diretoria; não ter tempo disponível e interesse para conhecer a proposta.

Percebeu-se que a rotina intensa das escolas, decorrentes da alta demanda de resolução de problemas, como a falta de professores, estrutura, indisciplina dos alunos etc., interferiu no envolvimento, tanto da participação de professores como na disponibilidade de material de suporte e espaço adequado para realizar as atividades. No entanto, apesar dessas limitações, muitas das diretorias/coordenações demonstraram interesse e curiosidade ao longo do processo, o que também foi observado com as alunas do NMB.

\section{ADESÃO}

Observou-se que, apesar de as alunas e diretorias apresentarem um grande interesse inicial pela proposta, a adesão não correspondeu ao esperado. Foi observado na fase da intervenção que 40,5\% (n=53) de um total de 131 participaram de mais de 10 sessões, e que $28,2 \%(n=37)$ nunca compareceram ao programa. A adesão foi ainda menor na fase de manutenção, em que somente $31,1 \%$ $(n=32)$ foram ao menos em 5 sessões.

Alguns pontos podem justificar a baixa adesão. Observou-se que, apesar de as alunas pertencerem a níveis socioeconômicos semelhantes (classe média-baixa), a localização da escola e a procedência das alunas (se moravam em comunidades ou não) determinavam maior ou menor adesão. Houve também variação entre as escolas quanto aos níveis de compreensão, atenção e disciplina das alunas. As escolas cujas adolescentes procediam de comunidades e áreas invadidas apresentaram estudantes de menor adesão, as quais apresentavam maiores dificuldades para acompanhar as atividades - com deficiências de leitura e atenção; também apresentavam uma frequência inconstante e falta de comprometimento com o programa. 
Nas escolas públicas, o fator socioeconômico, aliado a alguns determinantes do desempenho escolar, pode ter impacto no acompanhamento das atividades e, consequentemente, nas decisões quanto às escolhas alimentares e à prática de atividade física. Um relatório apresentado pelo Instituto Nacional de Estudos e Pesquisas Educacionais Anísio Teixeira (Inep) ao Ministério da Educação sobre a qualidade do ensino oferecido na escola pública verificou que somente $3 \%$ dos alunos de $8^{\mathrm{a}}$ série apresentavam um nível adequado de aprendizado, o que compromete o acompanhamento do conteúdo escolar ${ }^{24}$.

Análises conclusivas do relatório quanto aos principais determinantes indicam que, apesar da procedência das famílias ser importante no desempenho, fatores como a gestão adequada das escolas e o número de horas em sala de aula têm maior impacto no aprendizado ${ }^{24}$. No NMB, observou-se que as escolas com melhores gestões foram as que tiveram as maiores adesões ao programa.

A participação da família também é outro ponto a ser considerado na adesão. Observou-se uma baixa participação dos pais/responsáveis no evento de confraternização que tinha por objetivo oferecer vivências das atividades do programa. Os pais são considerados modelos para seus filhos em relação à saúde, no entanto a motivação e manutenção da proatividade desses pais nas intervenções ainda é um desafio. Estudos realizados em países de baixa/média renda demonstram que as famílias normalmente estão mais envolvidas na resolução dos problemas da rotina diária de seus filhos, relatam não ter tempo para participar das atividades promovidas pela escola e demonstram resistência em serem orientados pela escola ${ }^{19}$. Estudos de revisão sobre intervenções escolares indicam o uso de métodos diretos (aulas expositivas ou workshops presenciais) ao invés de indiretos (folhetos informativos e tarefas), como a forma mais efetiva de sensibilizar e envolver os pais no ambiente escolar e na melhora da relação com os filhos 25,26 .

Apesar das dificuldades observadas em relação à adesão ao programa, pode-se notar, no delineamento do NMB, potenciais determinantes da permanência das alunas no programa. Entre eles, pode-se citar o oferecimento dos lanches no intervalo entre o término das aulas curriculares e o início do programa. A inclusão do lanche ocorreu, pois, no pré-teste, muitas das alunas que voltavam para suas casas para almoçar não retornavam no período vespertino para participar do programa. Essa ação foi vista de forma positiva pelas alunas, pois o compartilhamento das refeições permitiu a discussão sobre opções saudáveis de alimentação, o contato com novos alimentos e gerou um senso de coletividade e o estimulo à participação no programa.

Outro ponto que teve efeito positivo na adesão foi a forma como a atividade física foi oferecida no programa. As atividades diversificadas, como danças, ioga, pilates, capoeira, jogos cooperativos, aeróbica, circuito, realizadas com o grupo, tinham como objetivo promover a diversão, o prazer na prática, e não na competição, respeitando a capacidade e habilidade individual das alunas.

\section{ESTRUTURA/MATERIAIS}

Em relação à estrutura para a prática das atividades, é importante que exista flexibilidade e criatividade na escolha de espaços adequados. Muitas das aulas foram adaptadas para serem realizadas nas salas de aula, por indisponibilidade de uso de quadras. No desenvolvimento de propostas fora do horário escolar, deve-se considerar a limitação de espaços, por conta da prioridade de uso destes para as práticas das aulas curriculares. Quanto aos materiais, soluções alternativas podem ser utilizadas para sanar as deficiências evidentes das escolas, com o uso de materiais de baixo, custo como bexigas, bolas feitas de meia calcas usadas, sacos de 
alimentos que podem ser utilizados como peso, e ainda com a aquisição de materiais não usuais, como petecas, espaguetes de piscina, frisbee, raquetes de praia.

\section{USO DAS TÉCNICAS INOVADORAS}

Intervenções que são baseadas em teorias, como a $\mathrm{TSC}^{27}$, são promissoras, por apresentarem maior impacto na mudança de comportamentos, do que as somente educacionais ${ }^{10,19}$. Estudos de prevenção de obesidade são pouco conclusivos sobre o que realmente é efetivo. É consenso na literatura que abordagens baseadas no controle de peso são ineficazes na perda e estabelecimento de um peso saudável, contribuindo para uma preocupação excessiva com a alimentação e corpo, a repetição de ciclos de perda e ganho de peso, a perda do foco na saúde, diminuição da autoestima, TA, estigmatização e discriminação corporal ${ }^{28,29}$.

Temas do NMB, como a aceitação corporal, influência da mídia, padrões de beleza, de não dicotomização dos alimentos entre bons e ruins, os riscos da restrição alimentar, deveriam ser incorporados em intervenções futuras por trazerem efeitos positivos, como a melhora da autoestima, da relação com a alimentação, satisfação corporal e redução dos comportamentos inadequados de controle de peso ${ }^{6}$

$\mathrm{O}$ uso da técnica da Entrevista Motivacional, testada pela primeira vez em um ambiente escolar brasileiro, apresenta-se como promissora, uma vez que permite trabalhar preocupações individuais e as barreiras para a mudança de comportamento ${ }^{11}$ - que muitas vezes não são acessadas em discussões de grupo. A resposta ao uso desta técnica foi heterogênea: muitas demonstravam motivação evidente para participar; enquanto outras, mesmo sendo mais introspectivas e com dificuldade de expressar seus pensamentos, aderiram as entrevistas. Percebeu-se que as alunas que estavam mais motivadas para participar do programa também estavam para participar das entrevistas, mas que, independentemente da motivação de cada uma delas, a maioria teve dificuldade em determinar metas relacionadas ao programa em suas vidas.

Ademais, das questões elencadas por tópicos anteriormente, deve-se questionar o próprio programa uma vez que vários desafios foram encontrados em sua implantação e que resultados significativos não foram encontrado $^{23}$. De qualquer forma, o NMB original gerou 26 publicações (disponível em www. newmovesonline.com) e foi reconhecido pelo Institute of Cancer como um programa de intervenção referência que produziu resultados comportamentais e psicossociais positivos.

Vale ressaltar que, apesar de o programa original ter sido bem avaliado, uma avaliação da sustentabilidade do programa nas escolas por professores, diretores e coordenadores, alguns anos depois, indicou que somente o componente relacionado com atividade física ('Seja Ativa') se manteve na maioria das escolas ${ }^{30}$ e que a continuidade das atividades ocorreu por estarem incluídas no currículo, a escola se preocupar com a saúde dos alunos e ter recursos financeiros, infraestrutura e equipe disponível para a aplicação do programa, questões também levantadas durante a implementação do NMB.

$O$ processo de adaptação em si seguiu todos parâmetros e cuidados recomendados de tradução e adequação à cultura e situação local, no entanto, poderia ser criticado quanto ao conteúdo excessivo de informações do manual, de alguns protocolos de dinâmicas por vezes tendo que ser modificados para atender limitações de tempo de intervenção e intercorrências decorrentes da desatenção e desinteresse das alunas, não sendo possível ser fiel na adaptação do original. Assim como no original, pode-se observar que o conteúdo relacionado com a atividade física foi o que teve maior aceitação, sendo considerado um modelo promissor a ser adotado em escolas. Os de suporte social e de nutrição devem ser reavaliados 
quanto à quantidade de informações disponibilizadas, podendo ser reduzidos em seu conteúdo e revistos quanto ao formato das dinâmicas utilizadas.

Consideradas essas questões, vale uma reflexão crítica sobre a adoção de programas desenvolvidos internacionalmente para aplicação no contexto em questão - mesmo que adaptados. Além dos aspectos alimentares e socioeconômicos, uma série de varáveis psicossocioculturais da cultura americana versus a cultura brasileira poderiam ser aprofundadas para a compressão das dificuldades de sucesso com o programa no cenário nacional. Novamente, uma delas é a realidade dos programas e estruturas escolares. Não é comum no Brasil que programas extracurriculares sejam abrangidos pelas escolas e que os alunos recebam e tenham familiaridade com tais programas. Assim, as escolas podem ser menos abertas e, inclusive, ter menos infraestrutura e oferecer menos apoio para a realização e adesão dos educandos. Alunos que já podem ter dificuldades com a estrutura padrão dos currículos podem ser desmotivados para se engajar em outras atividades 'pensantes' que pedem escrita, reflexão etc. Ainda na estrutura educacional americana clássica, os pais são mais engajados com as escolas de suas comunidades - inclusive os filhos classicamente estudam em escolas do bairro em que moram, e há outro senso de comunidade -, o que pode fazer diferença na participação dos pais e incentivo destes para adesão dos filhos.

Também é importante entender a problemática da segurança alimentar e básica nas esferas macro e microssocial no Brasil ${ }^{31}$, que difere dos Estados Unidos, como possível interferência nos resultados do programa. As escolhas alimentares, assim como a necessidade da prática de atividade física, e o autocuidado, nesse contexto, deixam de ter importância em vista das condições de risco maiores que envolvem a própria sobrevivência e impedem a reflexão e adoção de hábitos saudáveis. Nesse sentido, nas adolescentes do NMB, fica evidente que os temas tratados no programa estavam, por vezes, distantes da realidade social e que apesar do interesse inicial pelo programa estar associado a saúde, a manutenção no programa foi devido à atenção e ao acolhimento por parte dos profissionais de saúde.

Assim, as dificuldades enfrentadas na aplicação de uma intervenção podem ser desafiadoras, e deve-se pensar até se um programa internacional é 'adaptável' e aplicável. De qualquer forma, soluções podem ser encontradas por equipes comprometidas, bem formadas e bem coordenadas. É importante ressaltar a necessidade do treinamento dos profissionais da área da saúde na condução de um programa baseado na TSC. O nutricionista deve ir além do modelo tradicional de educação nutricional, no qual os conteúdos - direcionados à ciência dos alimentos - são passados de forma didática, com pouca interação com o público; e sim adquirir experiência em aplicação de dinâmicas de grupo, interagindo e conduzindo reflexões sobre os conteúdos. No NMB, é essencial que o nutricionista saiba transitar em discussões sobre os sentimentos, crenças, e percepções em relação à alimentação e às condições sociais envolvidas nas escolhas alimentares. Também deve promover um ambiente de não julgamento e aceitação, conduzindo os temas para a reflexão. Em relação às entrevistas individuais, é importante que o nutricionista seja treinado na técnica da entrevista motivacional, entendendo a sua dinâmica de aplicação, buscando desenvolver o estilo e as habilidades para condução da conversa. No entanto, mesmo com todos esses cuidados, esse formato pode ser desafiador e 'estranho' para os participantes (de escolas públicas) não acostumados ao formato.

Os educadores físicos devem ter experiência em práticas de atividade física em grupo, não somente na orientação da prática em si, mas também na interação com o grupo e com o indivíduo, reforçando as habilidades e minimizando as dificuldades. Dessa 
forma, ele incentiva a inclusão e participação de indivíduos normalmente não motivados a se interessarem pela prática de atividade física, entendendo que ela pode ser feita por prazer e diversão, e não somente por obrigação. Todavia, para atividades mais práticas, como as físicas, as barreiras foram menores no NMB.

\section{Considerações finais}

É essencial que se descreva o processo de aplicação de programas internacionais quanto à sua adaptação transcultural e às dificuldades metodológicas de implementação. Diferentemente da realidade desse projeto, que teve financiamento, é evidente o escasso investimento em pesquisa no país, e, consequentemente, há carência de estudos nacionais que possam gerar reflexões sobre o desenvolvimento de intervenções. Os resultados sempre se remetem a estudos internacionais, que diferem muito da realidade nacional em termos de tamanho de amostra, metodologia adotada e qualidade na análise dos dados, comprometendo a generalização de seus resultados, o que funcionaria em países de baixa-média renda, como o Brasil.

Observou-se que os desafios não se centram somente no conteúdo em si e no tipo de intervenção per si, mas que podem acontecer devido ao local, às questões relacionadas com as escolas (da estrutura à equipe) e ao perfil dos participantes. Os construtos desse programa são inovadores e um tanto inéditos e se mostraram 'incompreensíveis' - como o conceito de imagem corporal -, especialmente dado o nível sociocultural das participantes.

O NMB apresenta potencial para ser replicado e utilizado como referência para novos modelos de intervenção no país, sendo necessário adaptações nos seus conteúdos, com redução da quantidade de informações do manual, assim como na redução de atividades propostas em cada sessão, especificamente as relacionadas com o suporte social e alimentação, para que sejam mais bem compreendidos por populações com dificuldades de aprendizado e despertem o interesse nos temas abordados. Por ser considerado uma referência internacional, com muitos resultados positivos, pode ser usado como um modelo de estratégia comportamental diferenciado, podendo ser proposto em escolas de período integral como uma disciplina optativa direcionada às meninas.

\section{Colaboradores}

Karin Louise Lenz Dunker - Contribuiu substancialmente para a concepção e o planejamento ou para a análise e a interpretação dos dados; contribuiu significativamente na elaboração do rascunho ou revisão crítica do conteúdo e participou da aprovação da versão final do manuscrito.

Marle dos Santos Alvarenga - Contribuiu significativamente na elaboração do rascunho ou revisão crítica do conteúdo e participou da aprovação da versão final do manuscrito.

Angélica Medeiros Claudino - Contribuiu substancialmente para a concepção e para o planejamento ou para a análise e a interpretação dos dados.

\section{Agradecimentos}

Os autores agradecem a Dianne NeumarkSztainer, da University of Minnesota, por sua permissão para adaptar o programa New Moves, assim como suas considerações sobre o desenvolvimento desse projeto. 


\section{Referências}

1. Neumark-Sztainer D, Story M, Hannan PJ, et al. New Moves: a school-based obesity prevention program for adolescent girls. Prev Med 2003 Jul; 37(1):41-51.

2. Neumark-Sztainer D, Fried SE, Flattum CF, et al. New moves-preventing weight-related problems in adolescent girls: a group-randomized study. Am J Prev Med 2010 Nov; 39(5):421-432.

3. Neumark-Sztainer D, Story M, Hannan PJ, et al. Weight-related concerns and behaviors among overweight and non-overweight adolescents: Implications for preventing weight-related disorders. Arch Pediatr Adolesc Med 2002 Feb; 156(2):171-178.

4. Sherwood NE, Wall M, Neumark-Sztainer D, et al. Effect of socioeconomic status on weight change patterns in adolescents. Prev Chronic Dis 2009 Jan; 6(1):A19.

5. Shaw H, Stice E, Becker CB. Preventing eating disorders. Child Adolesc Psychiatr Clin N Am 2009 Jan; 18(1):199-207.

6. Ciao AC, Loth K, Neumark-Sztainer D. Preventing eating disorder pathology: common and unique features of successful eating disorders prevention programs. Curr Psychiatry Rep 2014 Jul; 16(7):1-13.

7. Khambalia A, Dickinson S, Hardy L, et al. A synthesis of existing systematic reviews and meta-analyses of school-based behavioural interventions for controlling and preventing obesity. Obes Rev 2012 Mar; 13(3):214-33.

8. Bandura A. Health promotion by social cognitive means. Health Educ Behav 2004 Apr; 31(2):143-164.

9. O’Dea J. Preventing body image problems: Schoolbased approaches. Encyclopedia of Body Image and Human Appearance. 2012; 686-92.

10. Cerin E, Barnett A, Baranowski T. Testing Theories of Dietary Behavior Change in Youth Using the Mediating Variable Model with Intervention Programs. J Nutr Educ Behav 2009 Sept-Oct, 41(5):309-318.

11. Miller WR, Rollnick S. Motivational Interviewing: Preparing People for Change. 2nd ed. New York: Guilford Press; 2002.

12. O'Halloran PD, Blackstock F, Shields N, et al. Motivational interviewing to increase physical activity in people with chronic health conditions: a systematic review and meta-analysis. Clin Rehabil 2014 Dec; 28(12):1159-71.

13. Resnicow K, McMaster F, Bocian A, et al. Motivational interviewing and dietary counseling for obesity in primary care: an RCT. Pediatrics 2015 Apr; 135(4):649-57.

14. Leme ACB, Lubans DR, Guerra PH, et al. Preventing obesity among Brazilian adolescent girls: Sixmonth outcomes of the Healthy Habits, Healthy Girls-Brazil school-based randomized controlled trial. Prev Med 2016 May; 86:77-83.

15. Cunha DB, De Souza BSN, Pereira RA, et al. Effectiveness of a randomized school-based intervention involving families and teachers to prevent excessive weight gain among adolescents in Brazil. Plos One 2013 Feb 25; 8(2):e57498.

16. Sichieri R, Trotte AP, Souza RA, et al. School randomised trial on prevention of excessive weight gain by discouraging students from drinking sodas. Public Health Nutr 2009 Feb; 12(2):197-202.

17. Vargas ICS, Sichieri R, Sandre-Pereira G, et al. Avaliação de programa de prevenção de obesidade em adolescentes de escolas públicas. Rev Saúde Públ 2011; 45(1):59-68.

18. Doak CM, Visscher TL, Renders CM, et al. The prevention of overweight and obesity in children and adolescents: a review of interventions and 
programmes. Obes Rev 2006 Feb; 7(1):111-136.

19. Verstraeten R, Roberfroid D, Lachat C, et al. Effectiveness of preventive school-based obesity interventions in low-and middle-income countries: a systematic review. Am J Clin Nutr 2012 Jul 3; 96(2):415-38.

20. Bull ER, Dombrowski SU, McClearly N, et al. Are interventions for low-income groups effective in changing healthy eating, physical activity and smoking behaviours? A systematic review and meta-analysis. BMJ Open. 2014 Nov 28; 4(11):e006046.

21. Burgess E, Hassmén $P$, Welvaert $M$, et al. Behavioural treatment strategies improve adherence to lifestyle intervention programmes in adults with obesity: a systematic review and meta-analysis. Clin Obes 2017 Apr; 7(2):105-114.

22. Gaglianone CP, Taddei JAAC, Colugnati FAB, et al. Nutrition education in public elementary schools of São Paulo, Brazil: the Reducing Risks of Illness and Death in Adulthood project. Rev Nutr 2006 May/ June; 19(3):309-20.

23. Dunker KLL, Claudino AM. Preventing weight-related problems among adolescent girls: A cluster randomized trial comparing the Brazilian 'New Moves' program versus observation. Obes.Res. Clin. Pract. 2018 Jan/Feb;12(1):102-115.

24. Menezes-Filho NA. Os determinantes do desempenho escolar do Brasil. Instituto Futuro Brasil, IBMEC, São Paulo; 2007 [acesso em 2018 mar 28]. Disponível em: https://www.todospelaeducacao. org.br/arquivos/biblioteca/f4e8070a-8390-479ca532-803bbf14993a.pdf.
25. Golan M, Crow S. Parents are key players in the prevention and treatment of weight-related problems. Nutr Rev 2004 Jan; 62(1):39-50.

26. Hingle MD, O'Connor TM, Dave JM, et al. Parental involvement in interventions to improve child dietary intake: a systematic review. Prev Med 2010 Aug; 51(2):103-11.

27. Abraham C, Michie S. A taxonomy of behavior change techniques used in interventions. Health Psychology 2008 May; 27(3):379-387.

28. Bacon L, Aphramor L. Weight Science: Evaluating the Evidence for a Paradigm Shift. Nutr J 2011 Jan $24 ; 10(1): 9-13$.

29. Sànchez-Carracedo D, Neumark-Sztainer D, López-Guimerà G. Integrated prevention of obesity and eating disorders: barriers, developments and opportunities. Public Health Nutr 2012 Dec; 15(12):2295-2309.

30. Friend S, Flattum CF, Simpson D, et al. The researchers have left the building: what contributes to sustaining schoolbased interventionsfollowing the conclusion of formal research support? J.Sch. Health. 2014 May; 84(5):326-33.

31. Freitas MCS, Pena PG. Segurança alimentar e nutricional: a produção do conhecimento com ênfase nos aspectos da cultura. Rev Nutr 2007 Jan/Fev; 20(1):69-81.

Recebido em 26/06/2017

Aprovado em 11/03/2018

Conflito de interesses: inexistente

Suporte financeiro: Fundação de Amparo à Pesquisa do Estado de São Paulo (Fapesp) (processo número: 2012/16952-8) e Conselho Nacional de Desenvolvimento Científico e Tecnológico (CNPq) (processo número: 483871/2013-3) 